\title{
RAS Equilibrium Involves in Mesenteric Lymph Return-Mediated Acute Kidney Injury in Mice Underwent Acute Hemorrhage
}

Yu-Jie Jin

Hebei North University

Kun Su

Hebei North University

Hong Zhang

Hebei North University

Jin Xie

Hebei North University

Hong Sun

Hebei North University

Niu-Niu Feng

Hebei North University

Hui-Bo Du

Hebei North University

Li-Min Zhang

Hebei North University

Wen-Li Zhang

North China University of Science and Technology

Xiu-Hong Yang

North China University of Science and Technology

Zi-Gang Zhao ( $\square$ zzghyl@126.com )

Hebei North University https://orcid.org/0000-0002-9434-5231

\section{Research article}

Keywords: Hemorrhagic shock, Acute kidney injury, Mesenteric lymph, Renin-angiotensin system, Angiotensin converting enzyme

Posted Date: July 9th, 2020

DOI: https://doi.org/10.21203/rs.3.rs-38715/v1 
License: (c) (i) This work is licensed under a Creative Commons Attribution 4.0 International License. Read Full License 


\section{Abstract}

Background: Previous studies demonstrated renin-angiotensin system (RAS) played vital roles in shockinduced organ injury, and mesenteric lymph return was involved in hemorrhagic shock-induced acute kidney injury (AKI). Nevertheless, whether RAS is involved in PHSML-mediated AKI remains unclear. Therefore, this study investigated the role of RAS in post-hemorrhagic shock mesenteric lymph (PHSML)induced AKI.

Methods: After acute hemorrhage and fluid resuscitation, the mice were treated with mesenteric lymph duct ligation (MLDL) and administrations of angiotensin converting enzyme (ACE) inhibitor enalapril, angiotensin (1-7) (Ang (1-7)), angiotensin II (Ang II) type 1 receptor (AT1R) inhibitor losartan, respectively. In addition, the parts of mice with hemorrhage plus MLDL were treated with Ang II, Mas receptor (MasR) inhibitor A-779, respectively. Meanwhile, the $A C E 2^{-/}$mice received hemorrhage plus MLDL. At $4 \mathrm{~h}$ after resuscitation, the kidneys were harvested for the observation of histomorphology and measurement of ACE, ACE2, AT1R, MasR expressions and Ang II and Ang (1-7) levels.

Results: Hemorrhagic shock induced renal tissue injury, increased the ACE and AT1R expressions, decreased the ACE2 and MasR expressions in kidney, accompanied by elevated Ang II and depressed Ang (1-7) in kidney. These adverse effects were partially reversed by MLDL or administrations of enalapril, Ang-(1-7), and losartan, respectively. In addition, the beneficial role of MLDL was reversed by ACE2 deficiency and Ang II or A-779 administrations.

Conclusion: MLDL alleviates hemorrhagic shock-induced AKI in mice is related to the equilibrium ACEAngll-AT1R and ACE2-Ang (1-7)-MasR axis.

\section{Background}

Hemorrhagic shock can result from a wide range of pathogenic factors, such as multiple trauma, peptic ulcer, intraoperative hemorrhage, is a common clinical intensive illness [1]. Acute kidney injury (AKI) is the common complications following hemorrhagic shock. Once patients occurred AKI, internal environment disorder aggravated, with high mortality rate [2]. Therefore, attention should be paid to the changes of renal function in the process of severe hemorrhagic shock. Recently, data from rat models indicate that post-hemorrhagic shock mesenteric lymph (PHSML) return is involved in hemorrhagic shock-induced AKI, and blockage of PHSML return alleviates AKI through reducing the inflammation and oxidative stress [3, 4]. Renin-angiotensin system (RAS) contains two main axes, as follows: angiotensin converting enzyme (ACE)-angiotensin (Ang) II-Ang II 1 receptor (AT1R) and ACE2-Ang (1-7)-Mas-related G-protein-coupled receptor (MasR). The activation of ACE-Ang II-AT1R increases the Ang II synthesis and leads to vasoconstriction, reabsorption of water and sodium and hypertension, even causes inflammation and fibrosis in renal tissue, which result in kidney injury. It is reported that Ang II leads to kidney injury according to AT1R and AT2R activating nuclear factor KB (NF-KB) signaling and pro-inflammatory genes, promoting the monocyte aggregation and the expression of pro-inflammatory cytokines [5]. ACE2-Ang 
(1-7)-MasR axis activation increases Ang (1-7) synthesis and causes the opposite results by acting on MasR. The imbalance of ACE/ACE2 is also involved in kidney injury in the tourniquet-induced ischemiareperfusion on hind limbs. And upregulated the expression of ACE2 improves the imbalance of ACE/ACE2 and alleviates kidney injury [6]. The kidney injury appears in the tourniquet-induced ischemia-reperfusion model after upregulated the expression of $\mathrm{ACE} / \mathrm{Ang}$ II in $\mathrm{ACE2^{-/- }}$ mice. However, it is still not clear that whether the imbalance of ACE/ACE2 is involved in the occurrence of hemorrhagic shock-induced AKI and whether PHSML return effects. Therefore, we regulated the balance of ACE/ACE2 according to using drugs related to ACE/ACE2 axis and gene knockout mice, to investigate the role of ACE/ACE2 balance in kidney injury mediated by PHSML.

\section{Methods}

\section{Materials}

Ang II (10271406) and Ang (1-7) (32GGAM12G1) were purchased from the Enzo (Farmingdale, NY). Enalapril (CDS020548) and Losartan Potassium (LRAA4718) were purchased from the Sigma-Aldrich (Louis, MO). A779 (P14701408) was purchased from the GenScript (Piscataway, NJ). Anti-ACE antibody (ab11734), Anti-ACE2 antibody (ab108252), and Anti-AT1R antibody (ab124505) were obtained from Abcam (Cambridge, MA). Anti-Mas1R antibody (AV51197) was purchased from the Sigma-Aldrich (St. Louis, MO). Mouse and Rabbit IgG-Immunohistochemical Kit SABC ready-to-use and DAB chromogenic kit were obtained from the Boster (Wuhan, China). BCA kit was purchased from the Solarbio (Beijing, China). ELISA kits of Ang II and Ang (1-7) were purchased from Huiying biological technology (Shanghai, China).

\section{Animals}

Health and male wild type C57 mice $(25 \pm 2 \mathrm{~g})$ were derived from the Academy of Military Medical Sciences (Beijing, China). ACE2 ${ }^{-/-}$mice were obtained from North China University of Science and Technology. After adaptive feeding, all mice were randomly divided into nine groups $(n=6)$. As follows: Sham, Shock, Shock + ligation, Shock + Enalapril, Shock + Losartan, Shock + Ang (1-7), Shock + ligation + $A C E 2^{-/}{ }^{-}$, Shock + ligation + Ang II, Shock + ligation + A-779. The mice were fasted for 12 hours and drank freely before the experiment. And all animal experiments were approved by the Animal ethics committee of Hebei North University.

\section{Hemorrhagic shock model}

Mice were suffered to acute hemorrhage [7], maintaining mean arterial pressure (MAP) of $40 \pm 2 \mathrm{mmHg}$ for 60 minutes, followed by resuscitation with shed whole blood and equal volume of ringer's solution. The same operation was performed without hemorrhage and resuscitation in the Sham group. Mesenteric lymph duct ligation (MLDL) was executed as soon as the end of resuscitation in the Shock + ligation, Shock + ligation + ACE2 ${ }^{-/-}$, Shock + ligation + Ang II, and Shock + ligation + A-779 groups. The 
administrations of ACE inhibitor Enalapril, Ang (1-7), AT1R inhibitor Losartan Potassium were performed with the doses of $10 \mathrm{mg} / \mathrm{kg}, 1 \times 10^{-7} \mathrm{~mol} / \mathrm{kg}, 50 \mathrm{mg} / \mathrm{kg}$ before resuscitation in the Shock + Enalapril, Shock + Ang (1-7), Shock + Losartan groups, respectively. In addition, the parts of mice with hemorrhage plus MLDL were treated with Ang II $\left(1 \times 10^{-7} \mathrm{~mol} / \mathrm{kg}\right)$, MasR inhibitor A-779 $(0.1 \mathrm{mg} / \mathrm{kg})$ before resuscitation, respectively. The murine kidneys were collected at 4 hours after resuscitation or corresponding time for further research. The left kidney in each mouse was used for histopathology observation and immunohistochemistry detection, and the right kidney is used for ELISA assay. Animals were sacrificed by vertebrae dislocation.

\section{Histopathology observation}

Left renal tissues were fixed in paraformaldehyde of $4 \%$. Subsequently, paraffin-embedded samples were cut into $4 \mu \mathrm{m}$ sections, conventionally deparaffinized and H\&E stained. Tissues were observed under the optical microscope.

\section{Immunohistochemistry assay}

Following the samples cut into $4 \mu \mathrm{m}$ sections, sections were differently incubated with the primary antibody (ACE 1:50; ACE2 1:200; AT1 1:400; Mas 1:200) and $4^{\circ} \mathrm{C}$ overnight. The sections subsequently incubated with a corresponding secondary antibody. Nuclei were stained with hematoxylin. Sections were observed under the optical microscope.

\section{ELISA assay}

The renal Ang II and Ang (1-7) levels were tested by ELISA kits according to the manufacturer. The protein was detected using BCA kits for the standardization. The optical density of each well was read at $450 \mathrm{~nm}$ using the multiscan spectrum (SpectraMax M3, Molecular Devices, USA).

\section{Data presentation and statistical analysis}

The data are presented as mean \pm SD (ELISA assay) or mean \pm SE (immunohistochemistry assay) and analyzed with SPSS 22.0 software. Each experiment was repeated at least three times. Difference among different groups was analyzed using one-way analyses of variance (ANOVA). Difference between two groups was analyzed using LSD test and SNK test. And the Tamhame'2 T2 test was used for the comparison among different group with heterogeneity of variance. Significance was defined as $P<0.05$.

\section{Results}

\section{Changes of renal tissue structure in mice after hemorrhagic shock}

Figure 1 showed that hemorrhagic shock significantly increased pathological injury in kidney. Compared with the sham group, mesangial cells were proliferation, basement membrane was ruptured, glomerular was compressed, renal tubular epithelial cells were high edema, even extending into vessel cavity and 
renal tubules were narrowing in kidney underwent acute hemorrhage and resuscitation. Compared with the shock group, shock + ligation group had lighter pathological changes. Mesangial cells were proliferation, but glomerular without compression, renal tubular epithelial cells were edema, and renal tubules were lightly narrowing and exfoliative protein of renal tubular epithelium was seen. Moreover, compared with the shock group, pathological changes were lighter, such as mesangial cells were lightly proliferation and renal tubular epithelial cells were lightly edema, balloon changes were occasionally seen in the shock + Enalapril, shock + Ang (1-7) and shock + Losartan groups. And the shock + ligation + $A C E 2^{-/-}$, shock + ligation + Ang II and shock + ligation + A-779 groups were more severe pathological changes compared to the shock + ligation group (Fig. 1).

\section{Changes of the levels of ACE, ACE2, AT1R and MasR in kidney of mice after hemorrhagic shock}

Compared with the sham group, the levels of ACE and AT1R in the kidney of mice were increased significantly in shock group (Fig. 2 and Fig. 3), along with the decreased expression levels of ACE2 and MasR (Fig. 4 and Fig. 5). Moreover, the levels of ACE, AT1R in the kidney of mice in the shock + Enalapril, shock + Ang (1-7) and shock + Losartan groups were decreased significantly compared to the shock group (Fig. 2 and Fig. 3), and the levels of ACE2, MasR in the shock + Enalapril, shock + Ang (1-7) and shock + Losartan groups were increased significantly compared to the shock group (Fig. 4 and Fig. 5). Furthermore, the ACE and AT1R levels in shock + ligation group were decreased significantly compared to the shock group (Fig. 2 and Fig. 3). While ACE2 level in shock + Ligation + $A C E 2^{-/-}$group was decreased significantly compared to the shock + ligation group (Fig. 4). And the MasR level in the shock + ligation + Ang II group was decreased compared to the shock + ligation group (Fig. 5). The renal expressions of ACE2 and MasR in the shock + ligation + A-779 group were decreased compared to the shock + ligation group (Fig. 4 and Fig. 5).

\section{Changes of the levels of Ang II and Ang (1-7) in kidney of mice after hemorrhagic shock}

The Ang II level in kidney of shock group was significantly enhanced than that in sham group, while the Ang (1-7) level was the opposite tendency. Moreover, the Ang II levels were remarkable lower in the shock + Enalapril, shock + Ang (1-7) and shock + Losartan groups than that in the shock group. The Ang $(1-7)$ levels in the shock + Ang (1-7) and shock + Losartan groups were observably increased compared to the shock group, and Ang (1-7) level in the shock + Enalapril group was slightly increased but with no statistically difference. Compared to shock + ligation group, the Ang II level in kidney in shock + ligation + Ang II group was significantly increased, but no statistically changes in the shock + Ligation $+A C E 2^{-/-}$ and shock + Ligation + A-779 groups. In addition, the Ang (1-7) content in the shock + ligation + ACE2 ${ }^{-/-}$ group was markedly decreased compared to the shock + ligation group. 


\section{Discussion}

The current study investigated the role of ACE/ACE2 in the process of PHSML mediated kidney injury on the basis of previous studies. It was found that RAS imbalance occurred in hemorrhagic shock mice, which is characterized by ACE-Ang II-AT1R axis upregulation and ACE2-Ang (1-7)-MasR axis downregulation. The inhibition of ACE-Ang II-AT1R axis and MLDL reduced the kidney injury through the RAS balance reestablishment. Conversely, the inhibition of ACE2-Ang (1-7)-MasR abolished the beneficial effect of MLDL alleviating kidney injury. These results suggested that the imbalance of RAS is involved in the kidney injury mediated by PHSML.

Histological observation showed that the renal injury in the shock group was more severe than that in the sham group. After the treatment with Enalapril, Ang (1-7) and Losartan, this injury was alleviated significantly. Meanwhile, MLDL alleviated the kidney injury of mice following hemorrhagic shock. And the intervention of Ang II, A-779 and ACE2 gene knockout abolished the beneficial effects of MLDL. The results showed that the pathology of kidney changed according to changing the balance of ACE/ACE2 in mice.

ACE is an important component of RAS, which is expressed in glomerular and tubular. The expression of ACE in kidney increased significantly and participated in the process of kidney injury in sepsis [8]. Our studies showed that hemorrhagic shock significantly increased the ACE and AT1R expressions and Ang level II in kidney, which were revered by the administrations of Enalapril, Losartan and Ang (1-7), respectively. These results indicate that changing the balance of ACE/ACE2 in mice could not only changes the expression of ACE in kidney, but also alleviates kidney injury by regulating the expressions of downstream receptors but the detailed mechanism remains to be further inquiried. Excessive Ang II leads to water-sodium retention, increased intraglomerular pressure, mesangial cells and mesangial matrix increased, causing renal function downregulation. The mechanism is related to the activation of AMPK/mTOR signaling, TLR4/NF-KB signaling and TGF- $\beta$ signaling [9-11].

ACE2 is a newly discovered carboxypeptidase in recent years, which converts Ang II into protective Ang (1-7), which acts on MasR to effect. Our data demonstrated that hemorrhagic shock significantly decreased the ACE2 expression in kidney, which was significantly increased by the administration of Enalapril, Losartan and Ang (1-7), accompanying by increased expressions of MasR and Ang (1-7). Previous studies have shown that Enalapril upregulate the ACE2 expression and downregulate the ACE expression in the heart of spontaneously hypertensive rats [12]. Klimas' study reported that Losartan upregulate the ACE2 and MasR expressions in the kidney obtained from spontaneously hypertensive rats [13]. These findings suggest that the above drugs can upregulate ACE2 and MasR expressions in the kidney, while downregulate the ACE expression. Its possible mechanism is inhibiting the activation of mitogen-activated protein kinase and NF-KB signalings [14, 15]. But its specific mechanism still needs to be further explored.

Previous experiments have confirmed that PHSML is one of the important factors of organs injury, and blocking PHSML return can reduce the organs injury [16-18]. In this study, the expressions of ACE and 
AT1R and Ang II level were decreased, while the expressions of ACE2 MasR and Ang (1-7) were increased in mesenteric lymphatic ligation group compared to the shock group. After administration of Ang II and MasR blocker A-779, the pathological changes of kidney in mesenteric lymphatic ligation group were significantly more severe than shock group, accompanied by the decreasing of ACE2 and MasR and increasing of $A C E$ and $A T 1 R$. In addition, $A C E 2^{-/-}$mice was more severe histopathological changes than that of mesenteric lymphatic ligation mice, even the lower expression of MasR and Ang (1-7). These results suggest that MLDL alleviates the imbalance of ACE/ACE2 and pathological changes in kidney, but the mechanism by which MLDL regulating the components in RAS was not clear.

Collectively, the current investigation showed that hemorrhagic shock induced the unbalance of ACE/ACE2 in murine kidneys and acute kidney injury, the treatments of ACE/Ang II/AT1R inhibition and MLDL alleviated PHSML-mediated kidney and recovered the balance of ACE/ACE2. These results indicate that ACE/ACE2 imbalance is involved in PHSML mediated kidney injury, which is beneficial to elucidate the mechanism of PHSML mediated kidney injury and explore the treatment of kidney injury after hemorrhagic shock in the future.

\section{Conclusion}

The imbalance of ACE/ACE2 is involved in the kidney injury mediated by PHSML. And RAS as a new target can regulate AKI after hemorrhagic shock.

\section{List Of Abbreviations}

RAS: renin-angiotensin system; PHSML: post-hemorrhagic shock mesenteric lymph; AKI: acute kidney injury; MLDL: mesenteric lymph duct ligation; MAP: mean arterial blood pressure; ACE: angiotensin converting enzyme; Ang-(1-7): angiotensin-(1-7); Ang II: angiotensin II; AT1R: Ang II type 1 receptor; MasR: Mas-related G-protein-coupled receptor; MAPK: mitogen-activated protein kinase; NF-kB: nuclear factor KB.

\section{Declarations}

\section{Ethics approval and consent to participate}

All experiments were performed in accordance with the guidelines of the Animal ethics committee of Hebei North University.

\section{Consent for publication}

Not applicable. 


\section{Availability of data and materials}

The datasets used and/or analyzed during the current study are available from the corresponding author on reasonable request.

\section{Competing interests}

Not applicable.

\section{Funding}

This work was supported by the Natural Science Foundation of Hebei Province (H2017405017). The investigators had full responsibility for the study design, data collection and analyze and the manuscript.

\section{Authors' contributions}

Study concept and design: Z-GZ. Performed and analyzed experiments: Y-JJ, KS, JX, HS, N-NF, H-BD, LMZ, W-LZ and X-HY. Manuscript draft and edits: HZ, Y-JJ and Z-GZ. All authors read and approved the final version of the manuscript.

\section{Acknowledgements}

We are indebted to Chun-Yu Niu (PhD, Hebei Medical University, Shijiazhuang, Hebei, China) for his guidance.

\section{References}

1. Xie Y, Barbosa ACS, Xu M, Oberly PJ, Ren S, Gibbs RB, Poloyac SM, Song WC, Fan J, Xie W: Hepatic Estrogen Sulfotransferase Distantly Sensitizes Mice to Hemorrhagic Shock-Induced Acute Lung Injury. Endocrinology 2020, 161(1).

2. Wu M, Luan YY, Lu JF, Li H, Zhan HC, Chen YH, Zhang F, Tian YY, Yang ZL, Yao YM et al: Platelet count as a new biomarker for acute kidney injury induced by hemorrhagic shock. Platelets 2020, 31(1):94-102.

3. Niu CY, Zhao ZG, Ye YL, Hou YL, Zhang YP: Mesenteric lymph duct ligation against renal injury in rats after hemorrhagic shock. Renal failure 2010, 32(5):584-591.

4. Zhao ZG, Zhang LM, Lv YZ, Si YH, Niu CY, Li JC: Changes in renal tissue proteome induced by mesenteric lymph drainage in rats after hemorrhagic shock with resuscitation. Shock 2014, 42(4):350-355. 
5. Alique M, Sanchez-Lopez E, Rayego-Mateos S, Egido J, Ortiz A, Ruiz-Ortega M: Angiotensin II, via angiotensin receptor type $1 /$ nuclear factor-kappaB activation, causes a synergistic effect on interleukin-1-beta-induced inflammatory responses in cultured mesangial cells. J Renin Angiotensin Aldosterone Syst 2015, 16(1):23-32.

6. Yang XH, Wang YH, Wang JJ, Liu YC, Deng W, Qin C, Gao JL, Zhang LY: Role of angiotensinconverting enzyme (ACE and ACE2) imbalance on tourniquet-induced remote kidney injury in a mouse hindlimb ischemia-reperfusion model. Peptides 2012, 36(1):60-70.

7. Zhang H, Zhai JY, Du HB, Zhang LM, Li LF, Bian AQ, Jiang LN, Zhao ZG: Mesenteric lymph drainage alleviates hemorrhagic shock-induced spleen injury and inflammation. Acta Cir Bras 2019, 34(9):e201900903.

8. Rice CL, Kohler JP, Casey L, Szidon JP, Daise M, Moss GS: Angiotensin-converting enzyme (ACE) in sepsis. Circ Shock 1983, 11(1):59-63.

9. Fujihara S, Morishita A, Ogawa K, Tadokoro T, Chiyo T, Kato K, Kobara H, Mori H, Iwama H, Masaki T: The angiotensin II type 1 receptor antagonist telmisartan inhibits cell proliferation and tumor growth of esophageal adenocarcinoma via the AMPKalpha/mTOR pathway in vitro and in vivo. Oncotarget 2017, 8(5):8536-8549.

10. Mehta T, Buzkova P, Kizer JR, Djousse L, Chonchol M, Mukamal KJ, Shlipak M, Ix JH, Jalal D: Higher plasma transforming growth factor (TGF)-beta is associated with kidney disease in older community dwelling adults. BMC Nephrol 2017, 18(1):98.

11. Zhao H, Zheng Q, Hu X, Shen H, Li F: Betulin attenuates kidney injury in septic rats through inhibiting TLR4/NF-kappaB signaling pathway. Life Sci 2016, 144:185-193.

12. Yang Z, Yu X, Cheng L, Miao LY, Li HX, Han LH, Jiang WP: Effects of enalapril on the expression of cardiac angiotensin-converting enzyme and angiotensin-converting enzyme 2 in spontaneously hypertensive rats. Arch Cardiovasc Dis 2013, 106(4):196-201.

13. Klimas J, Olvedy M, Ochodnicka-Mackovicova K, Kruzliak P, Cacanyiova S, Kristek F, Krenek P, Ochodnicky P: Perinatally administered losartan augments renal ACE2 expression but not cardiac or renal Mas receptor in spontaneously hypertensive rats. J Cell Mol Med 2015, 19(8):1965-1974.

14. Li Y, Cao Y, Zeng Z, Liang M, Xue Y, Xi C, Zhou M, Jiang W: Angiotensin-converting enzyme 2/angiotensin-(1-7)/Mas axis prevents lipopolysaccharide-induced apoptosis of pulmonary microvascular endothelial cells by inhibiting JNK/NF-kappaB pathways. Sci Rep 2015, 5:8209.

15. Li Y, Zeng Z, Li Y, Huang W, Zhou M, Zhang X, Jiang W: Angiotensin-converting enzyme inhibition attenuates lipopolysaccharide-induced lung injury by regulating the balance between angiotensinconverting enzyme and angiotensin-converting enzyme 2 and inhibiting mitogen-activated protein kinase activation. Shock 2015, 43(4):395-404.

16. Fanous MY, Phillips AJ, Windsor JA: Mesenteric lymph: the bridge to future management of critical illness. JOP 2007, 8(4):374-399.

17. Watkins AC, Caputo FJ, Badami C, Barlos D, Xu DZ, Lu Q, Feketeova E, Deitch EA: Mesenteric lymph duct ligation attenuates lung injury and neutrophil activation after intraperitoneal injection of 
endotoxin in rats. J Trauma 2008, 64(1):126-130.

18. Badami CD, Senthil M, Caputo FJ, Rupani BJ, Doucet D, Pisarenko V, Xu DZ, Lu Q, Feinman R, Deitch EA: Mesenteric lymph duct ligation improves survival in a lethal shock model. Shock 2008, $30(6): 680-685$.

\section{Figures}

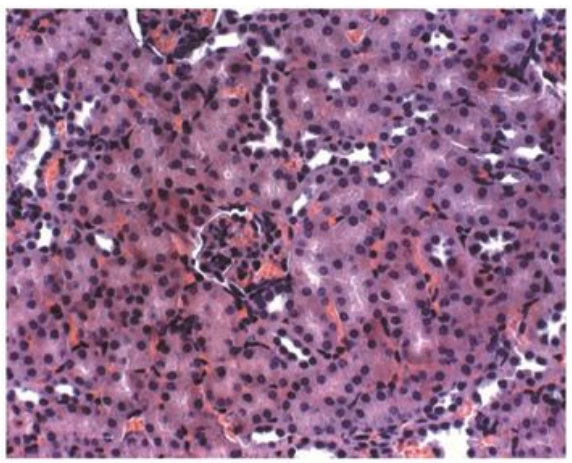

Sham

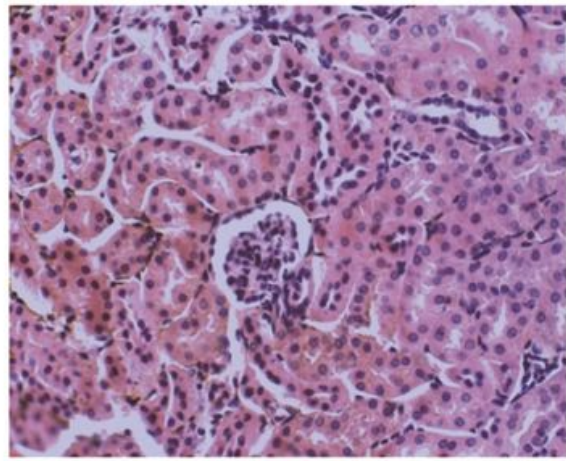

Shock+Enalapril

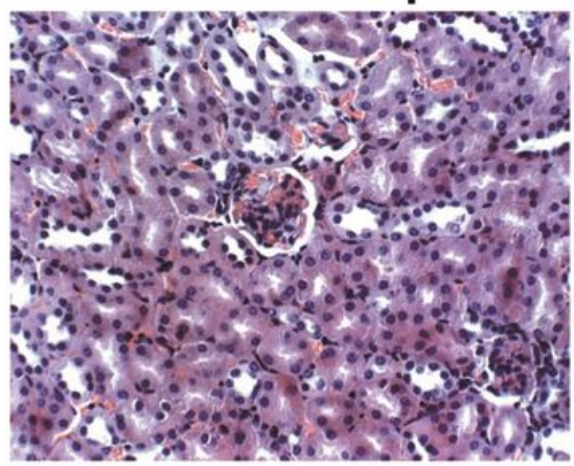

Shock+Ligation+ACE2-/-

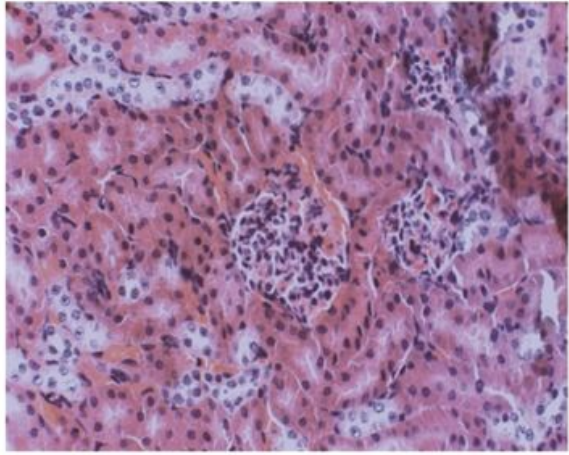

Shock

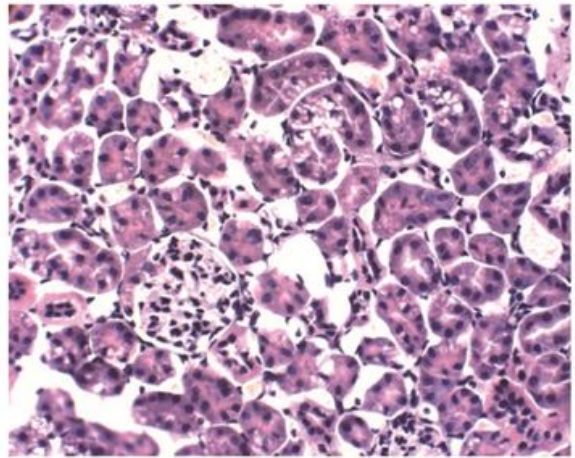

Shock+Ang (1-7)

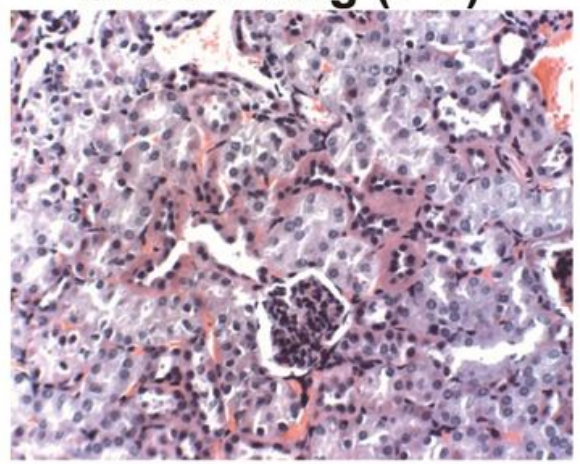

Shock+Ligation+Ang II

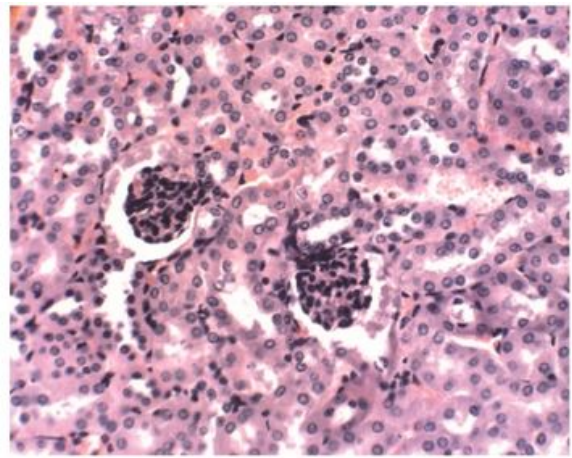

Shock+Ligation

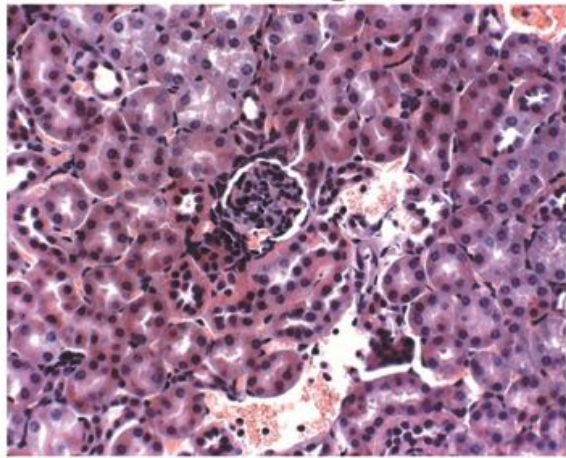

Shock+Losartan

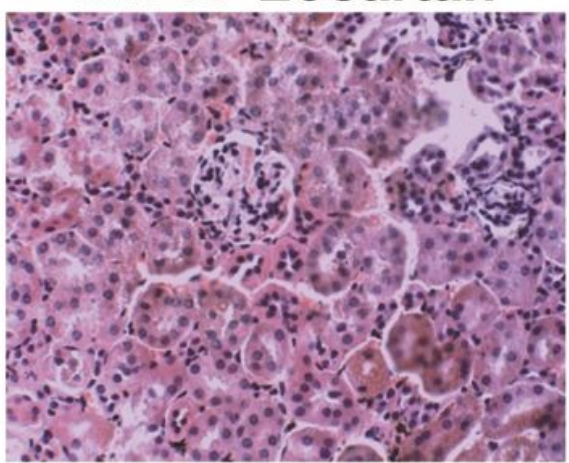

Shock+Ligation+A-779

Figure 1

Representative images of renal tissue obtained from mice following hemorrhagic shock (HE staining, $400 \times)$. 
A

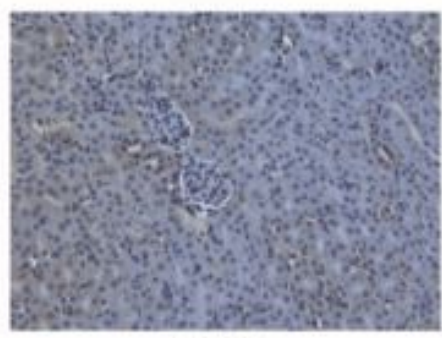

Sham

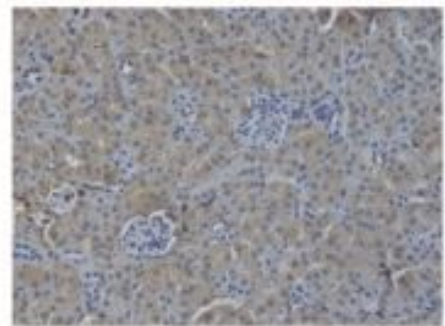

Shock+Enalapril

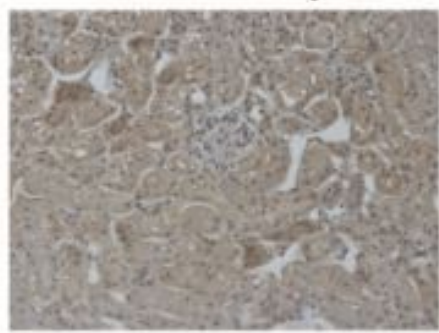

Shock+Ligation+ACE2-I-

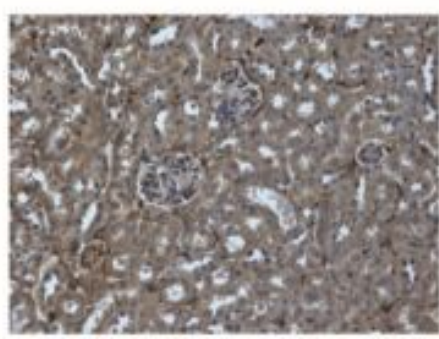

Shock

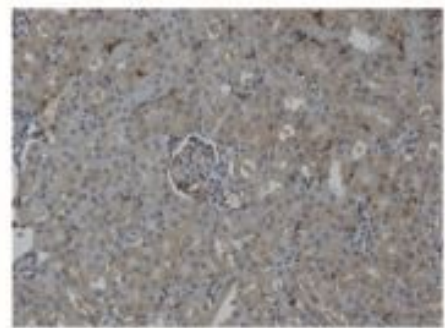

Shock+Ang (1-7)

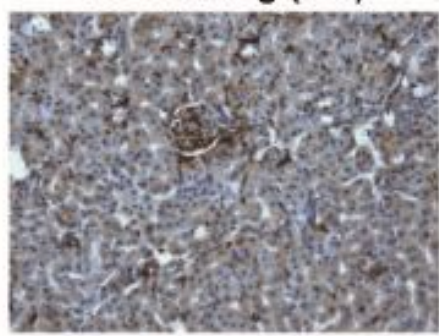

Shock+Ligation+Ang II

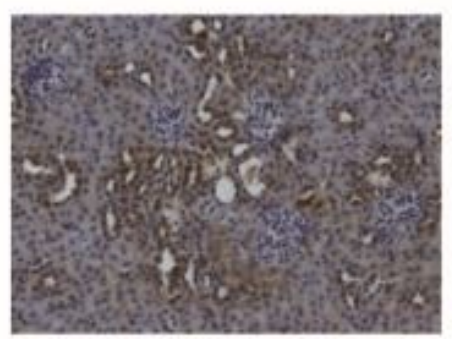

Shock+Ligation

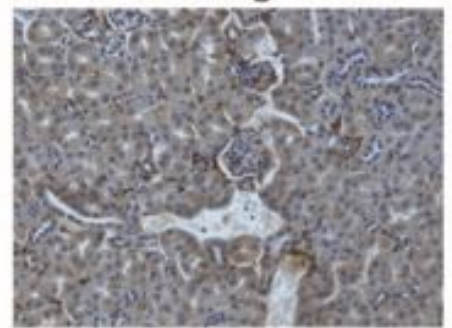

Shock+Losartan

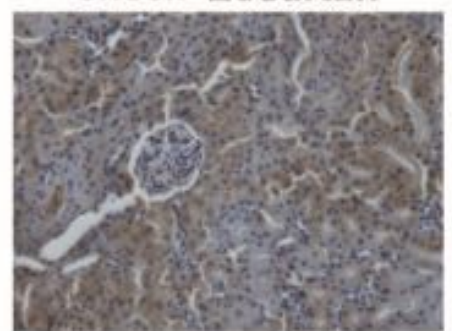

Shock+Ligation+A-779

\section{B}

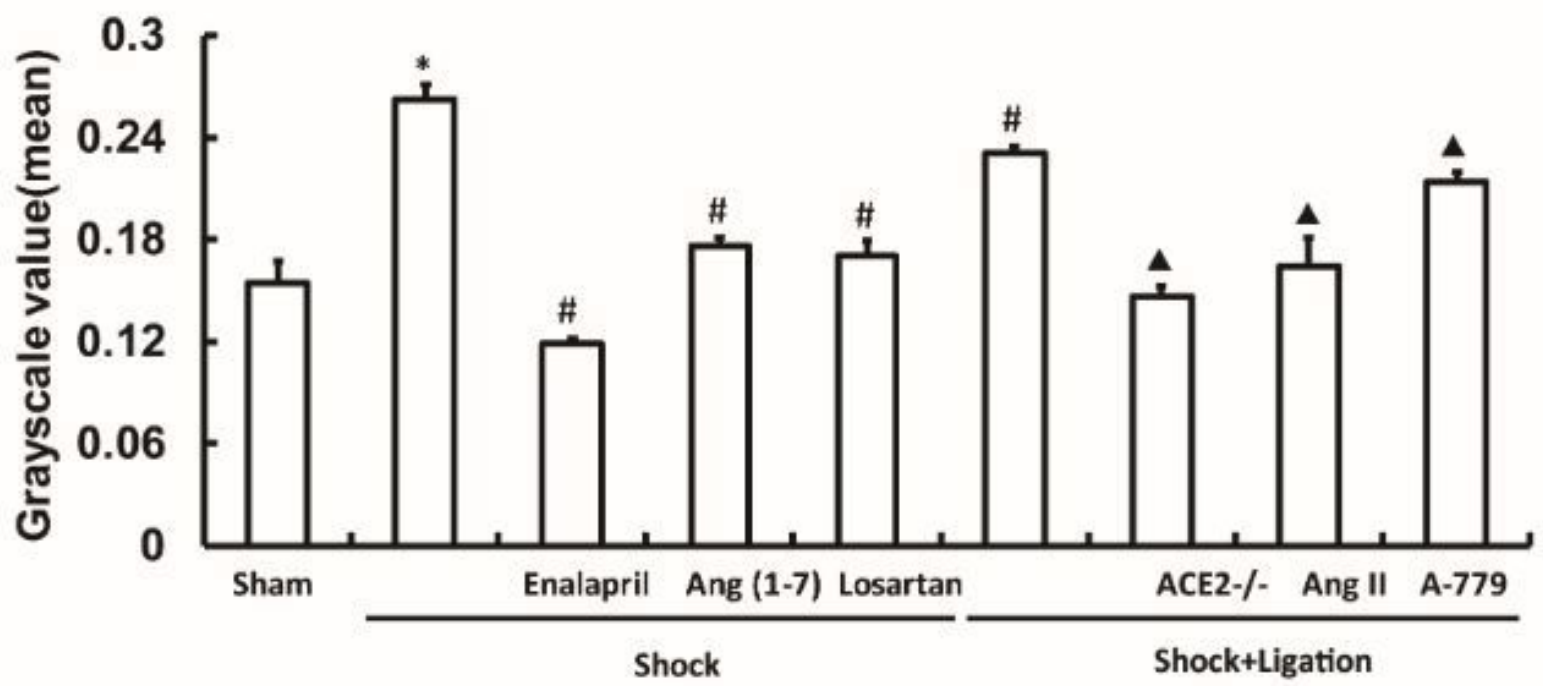

Figure 2

Changes of ACE expression in renal tissue of mice following hemorrhagic shock. A: Representative images of immunohistochemical staining (400x); B: Mean grayscale value of ACE expression in kidney, Data presented as the mean $\pm S E(n=3)$. ${ }^{*} P<0.05$, compared with the sham group; $\# P<0.05$, compared with the shock group; $\Delta \mathrm{P}<0.05$, compared with the shock+ligation group. 


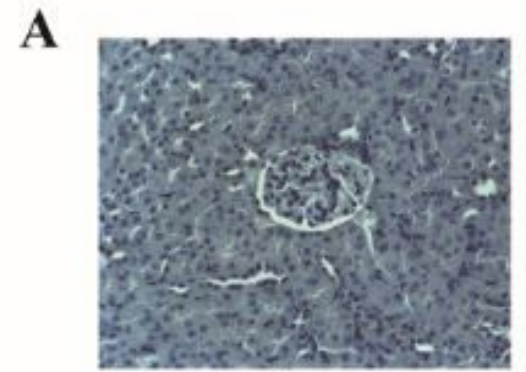

Sham

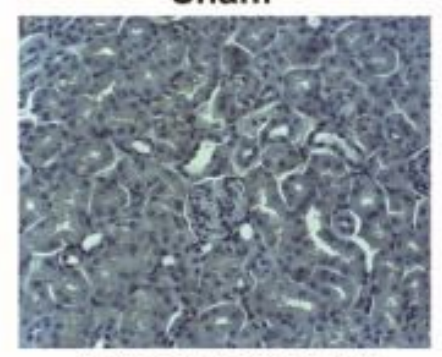

Shock+Enalapril

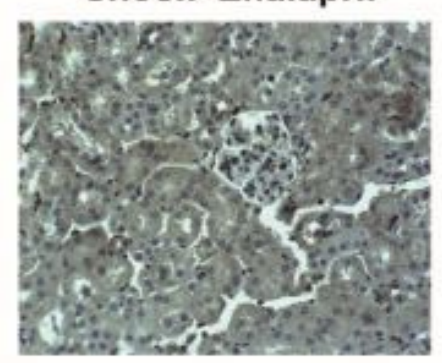

Shock+Ligation+ACE2-/-

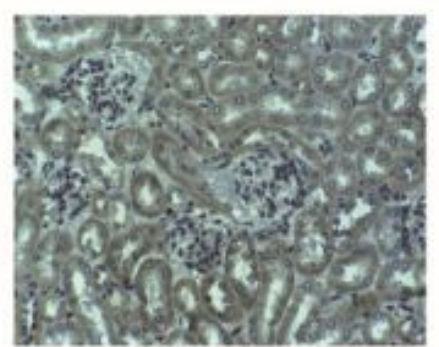

Shock

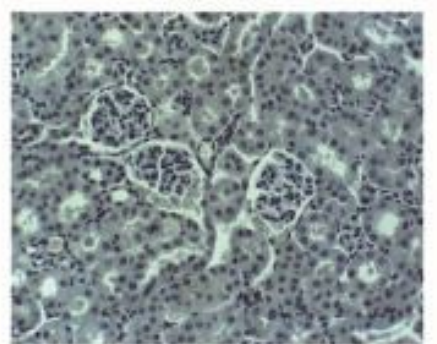

Shock+Ang (1-7)

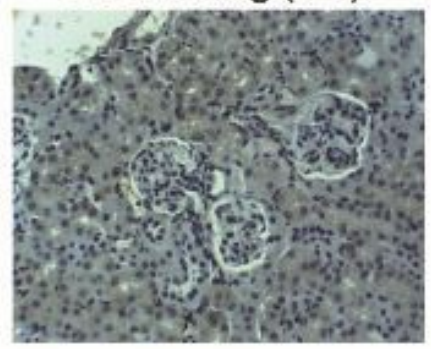

Shock+Ligation+Ang II

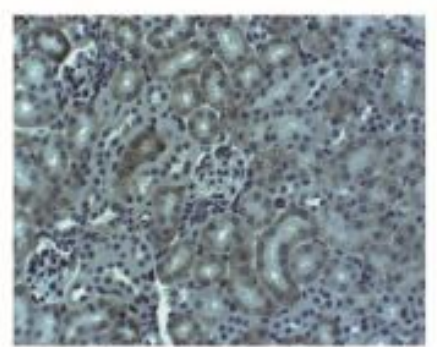

Shock+Ligation

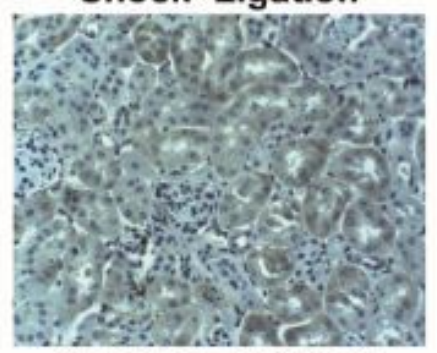

Shock+Losartan

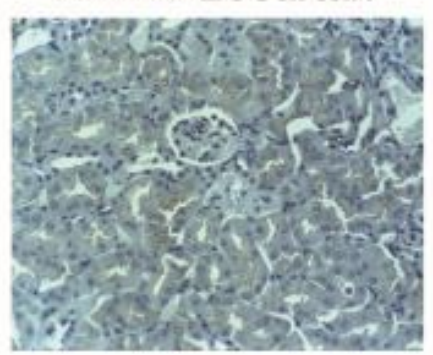

Shock+Ligation+A-779

B

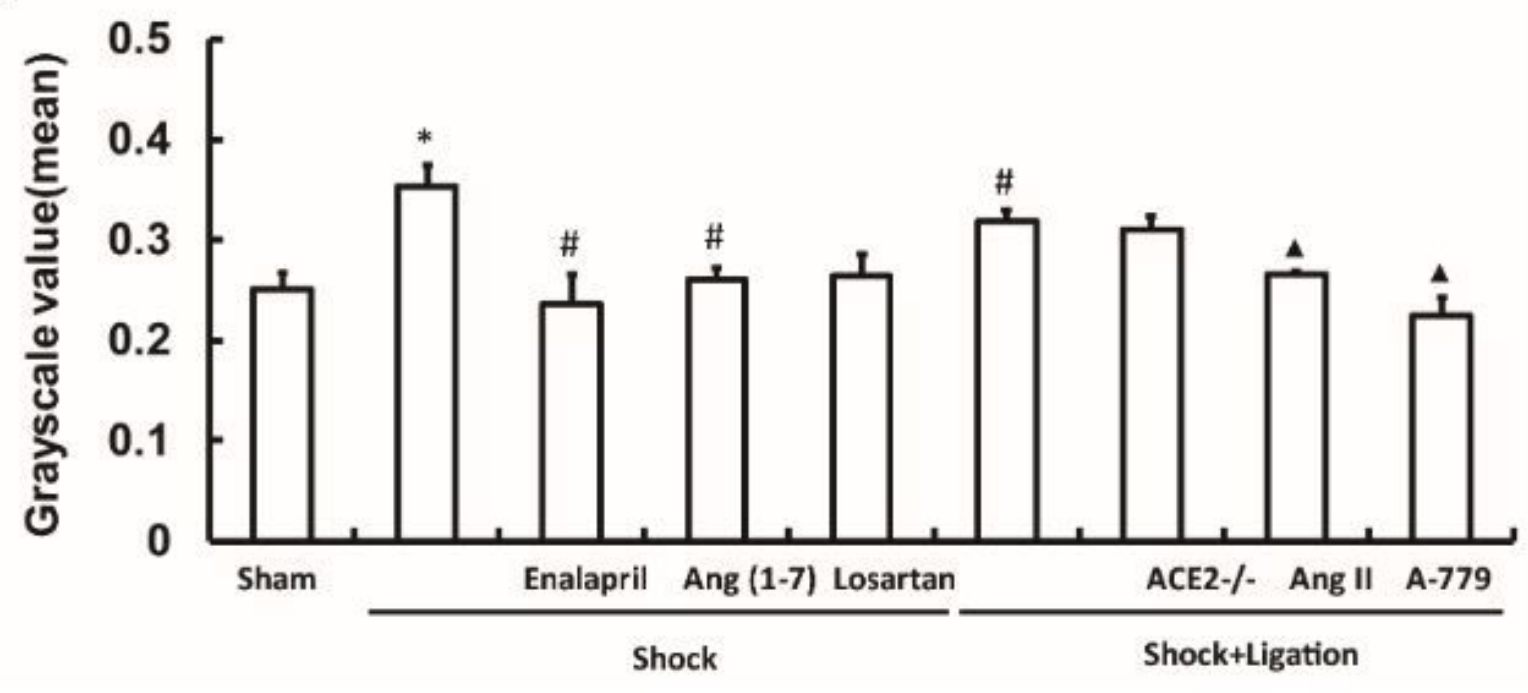

Figure 3

Changes of AT1R expression in renal tissue of mice following hemorrhagic shock. A: Representative images of immunohistochemical staining (400x); B: Mean grayscale value of AT1R expression in kidney, Data presented as the mean $\pm S E(n=3) .{ }^{*} P<0.05$, compared with the sham group; \#P<0.05, compared with the shock group; $\Delta \mathrm{P}<0.05$, compared with the shock+ligation group. 
A

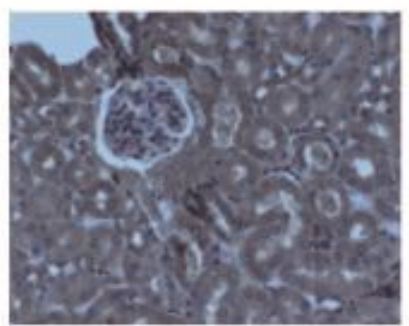

Sham

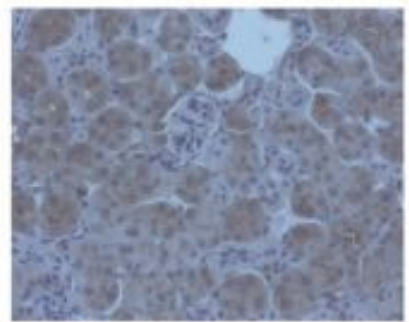

Shock+Enalapril

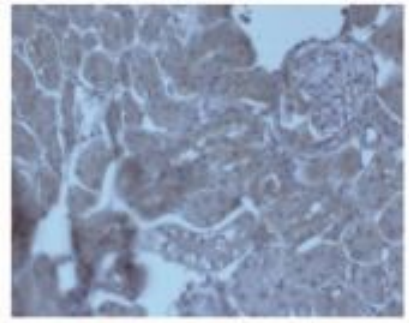

Shock+Ligation+ACE2-/-

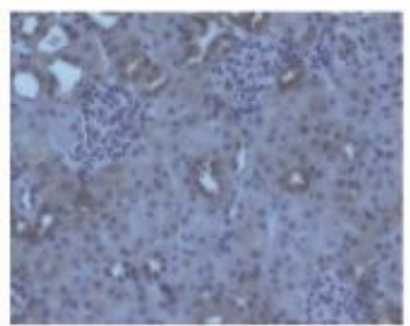

Shock

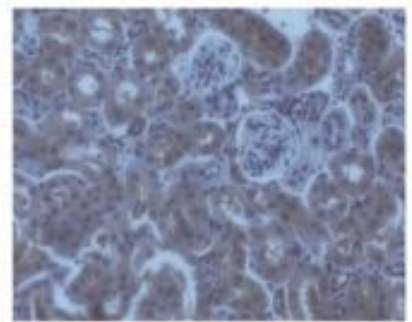

Shock+Ang (1-7)

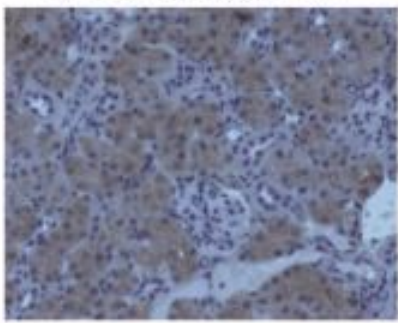

Shock+Ligation+Ang II

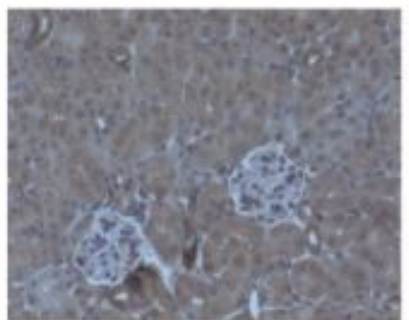

Shock+Ligation

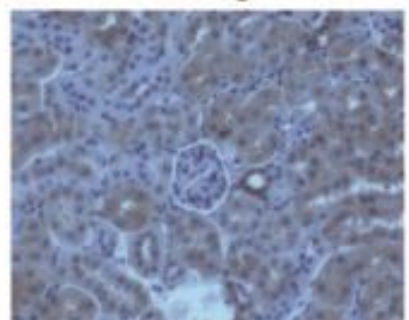

Shock+Losartan

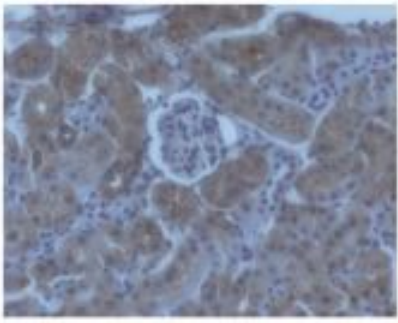

Shock+Ligation+A-779

B

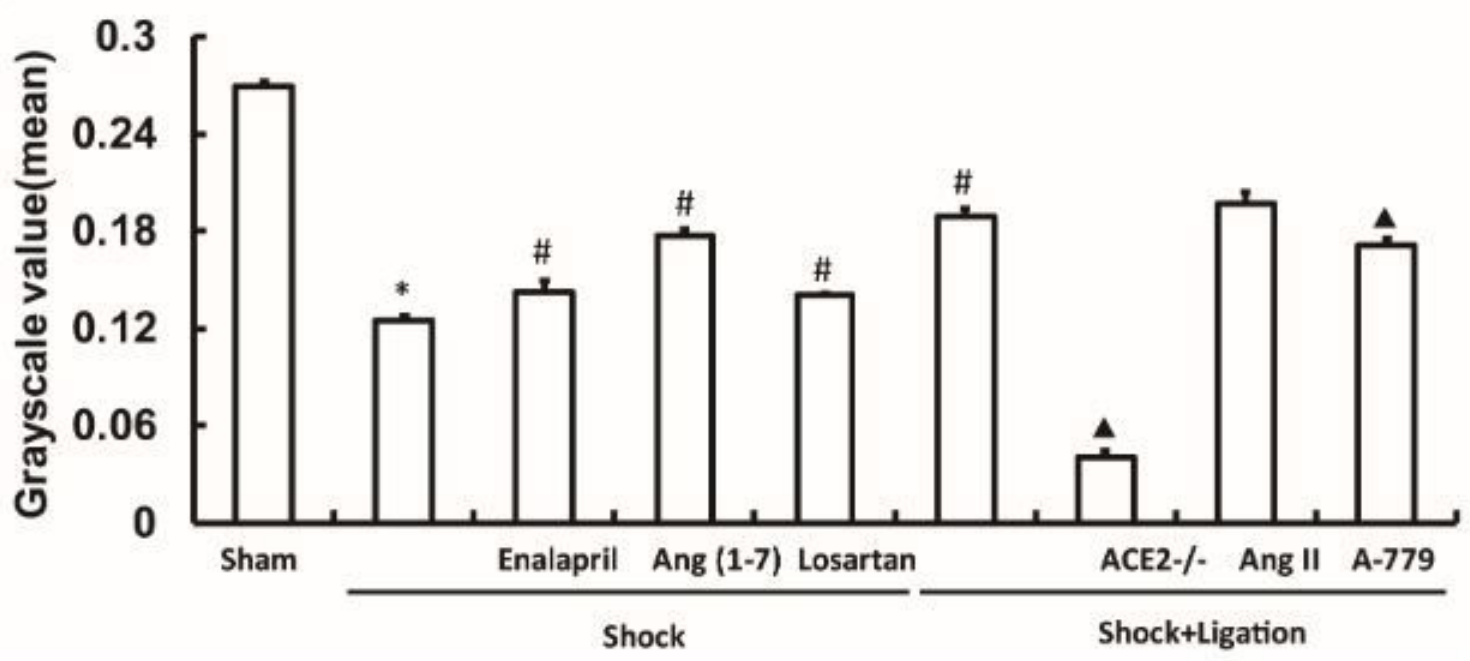

Figure 4

Changes of ACE2 expression in renal tissue of mice following hemorrhagic shock. A: Representative images of immunohistochemical staining (400x); B: Mean grayscale value of ACE2 expression in kidney, Data presented as the mean $\pm S E(n=3) .{ }^{*} P<0.05$, compared with the sham group; $\# P<0.05$, compared with the shock group; $\boldsymbol{\Delta}<<0.05$, compared with the shock+ligation group. 
A

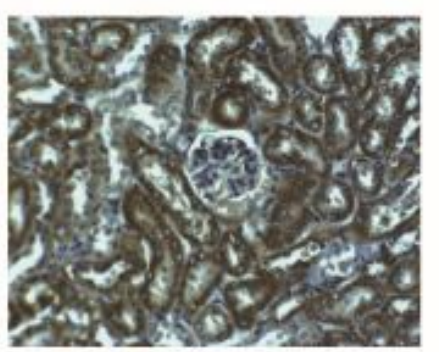

Sham

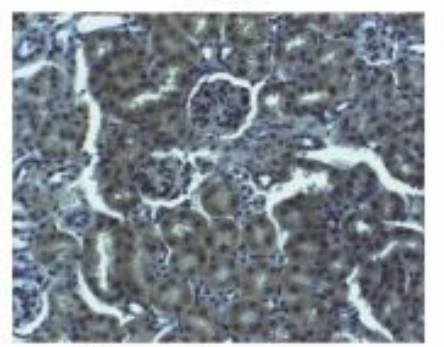

Shock+Enalapril

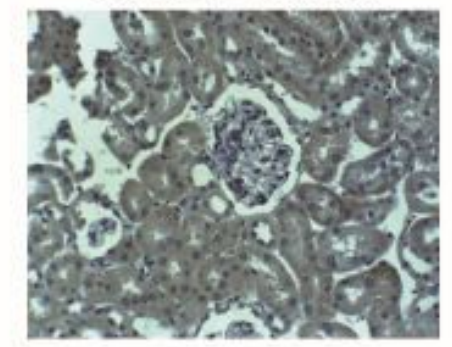

Shock+Ligation+ACE2-/-

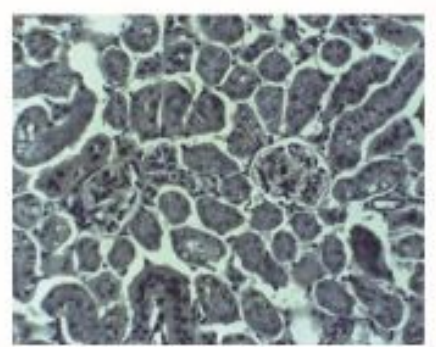

Shock

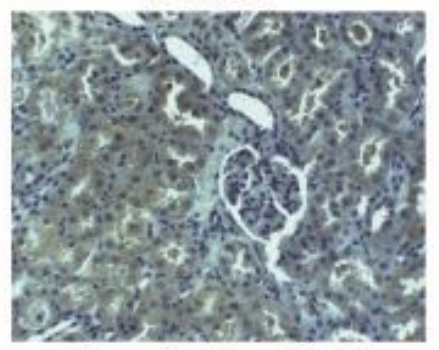

Shock+Ang (1-7)

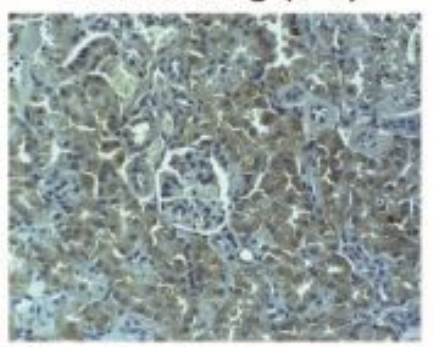

Shock+Ligation+Ang II

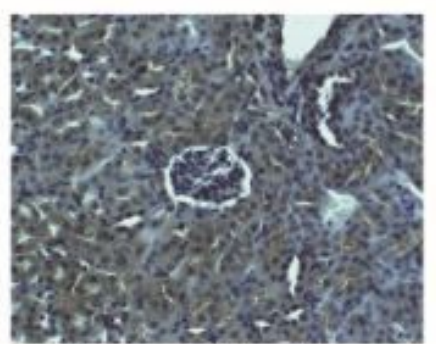

Shock+Ligation

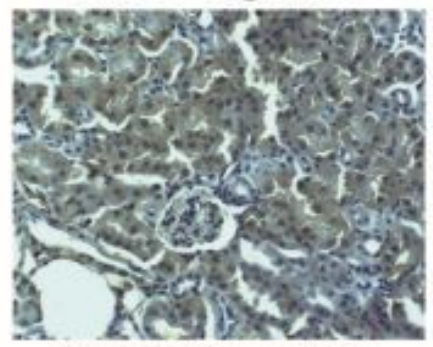

Shock+Losartan

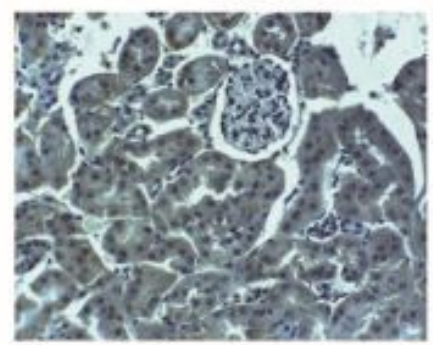

Shock+Ligation+A-779

\section{B}

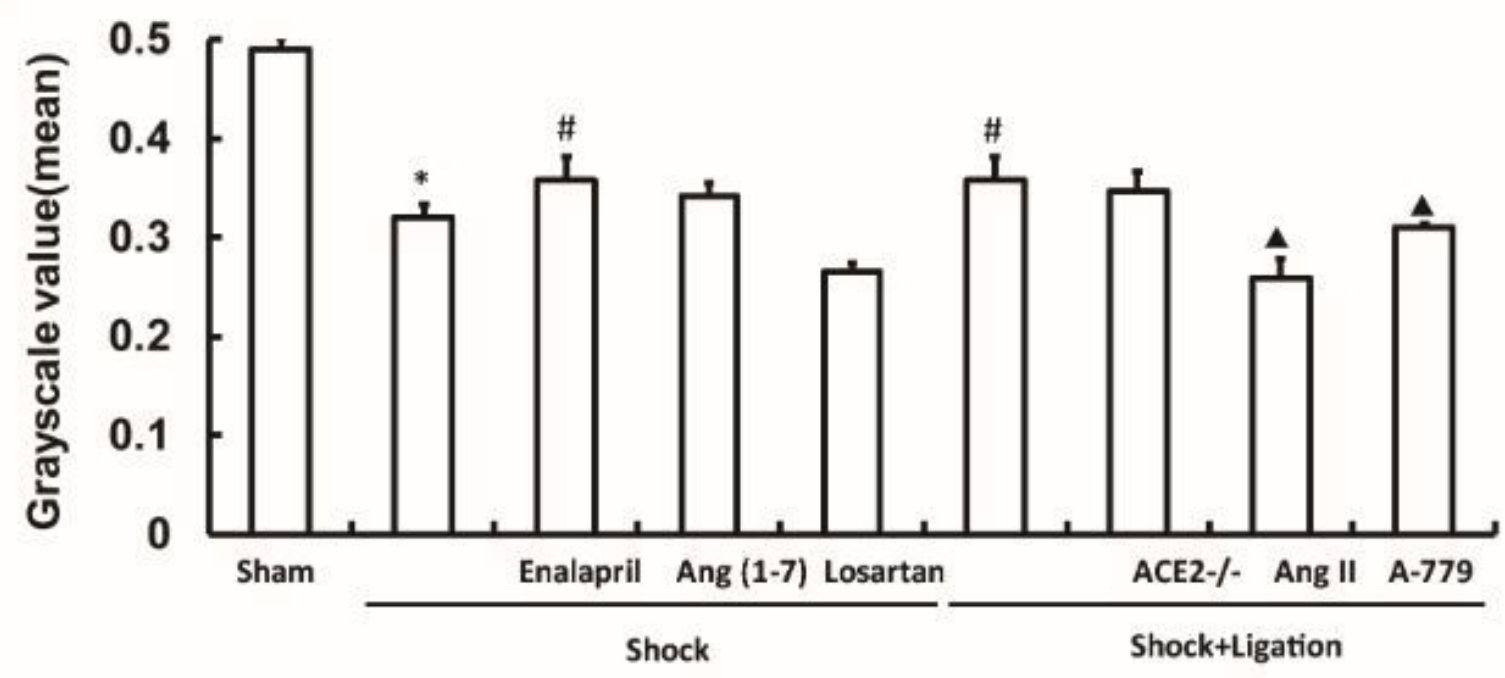

Figure 5

Changes of Mas receptor (MasR) expression in renal tissue of mice following hemorrhagic shock. A: Representative images of immunohistochemical staining (400x); B: Mean grayscale value of MasR expression in kidney, Data presented as the mean $\pm S E(n=3)$. $P<0.05$, compared with the sham group; \# $\mathrm{P}<0.05$, compared with the shock group; $\boldsymbol{\Delta} \mathrm{P}<0.05$, compared with the shock+ligation group. 
A
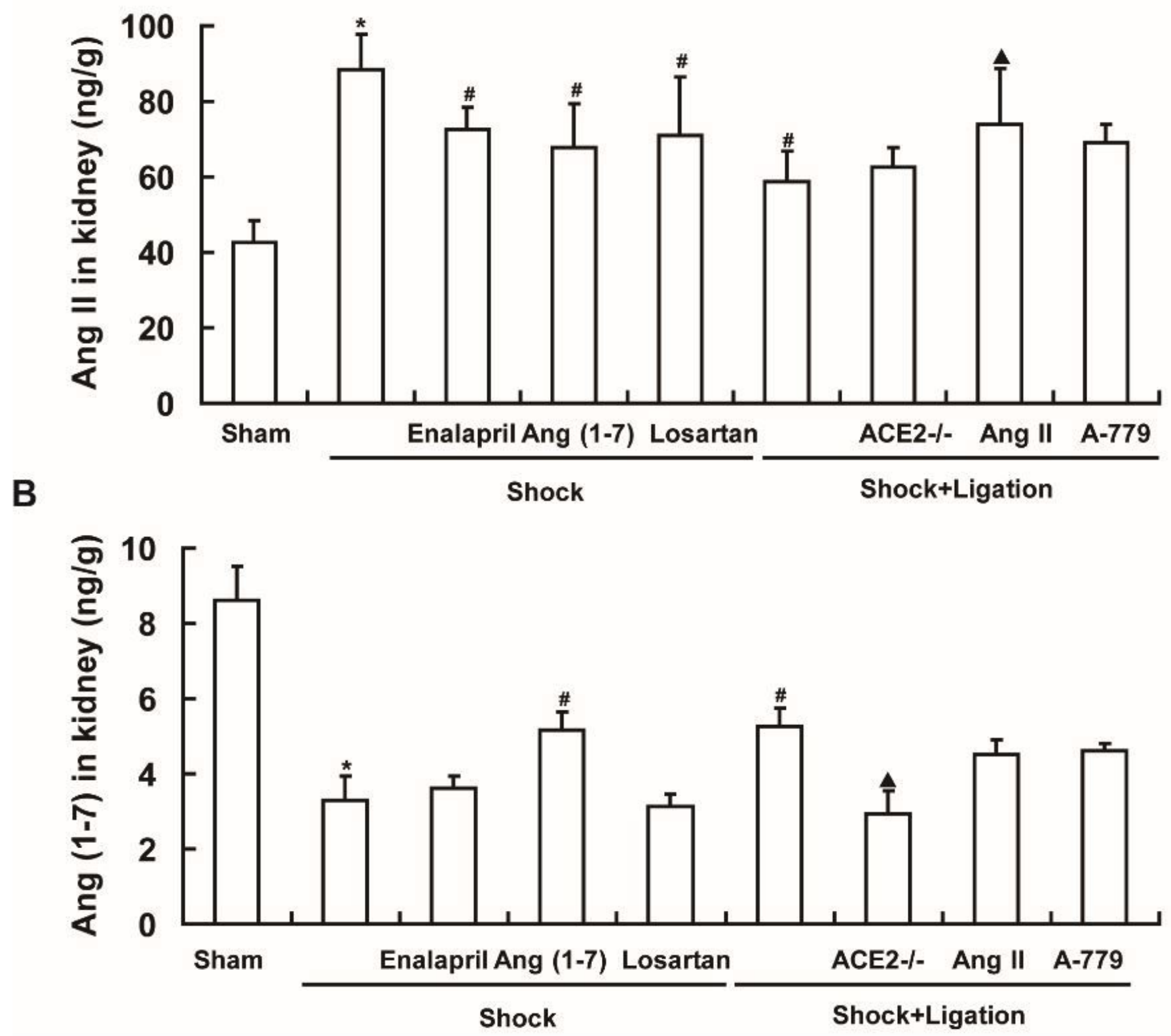

Figure 6

Changes of Ang II and Ang (1-7) in renal tissue of mice following hemorrhagic shock. Data presented as the mean $\pm S D(n=6)$. * $P<0.05$, compared with the sham group; \# $P<0.05$, compared with the shock group; $\boldsymbol{A}<0.05$, compared with the shock+ligation group.

\section{Supplementary Files}

This is a list of supplementary files associated with this preprint. Click to download.

- 0702NC3RsARRIVEGuidelinesChecklist2014.pdf 